\title{
Manequim de treinamento para o manejo do neonato com dispositivos implantados cirurgicamente: ConectNeo
}

\author{
Training manikin for neonate management with surgically implanted devices: ConectNeo \\ Maniquí de formación para el manejo neonato con dispositivos implantados quirúrgicamente: \\ ConectNeo
}

Recebido: 11/10/2021 | Revisado: 1610/2021 | Aceito: 17/10/2021 | Publicado: 20/10/2021

\author{
Ana Paula Aparecida dos Santos Varela \\ ORCID: https://orcid.org/0000-0002-7998-4190 \\ Universidade do Estado do Pará, Brasil \\ E-mail: dandaeu@hotmail.com \\ Edson Yuzur Yasojima \\ ORCID: https://orcid.org/0000-0002-1377-4895 \\ Universidade do Estado do Pará, Brasil \\ E-mail: yasojima@globo.com.br \\ Lêda Lima da Silva \\ ORCID: https://orcid.org/0000-0002-8729-2290 \\ Universidade do Estado do Pará, Brasil \\ E-mail: ledals500@gmail.com \\ Mariseth Carvalho de Andrade \\ ORCID: https://orcid.org/0000-0002-6257-5972 \\ Universidade do Estado do Pará, Brasil \\ E-mail: mariestat@outlook.com
}

\begin{abstract}
Resumo
Objetivo: desenvolver e validar um manequim neonatal para treinamento de habilidades no manuseio do neonato com dispositivos invasivos implantados cirurgicamente. Método: trata-se de uma pesquisa quantitativa descritiva, com participação de 21 especialistas no processo de avaliação do manequim para validação, respeitando todos os aspectos éticos que envolvem pesquisa desse cunho. Para avaliação do valor didático do produto desenvolvido foi proposto um teste de validação, consistindo em experimentação do modelo, aplicação de um questionário autoinstrutivo contendo questões sociodemográficas e dez escalas tipo Likert, que foram adaptadas para cada dispositivo avaliado. Resultados e discussão: foram avaliados 10 dispositivos no modelo completo sendo eles: derivação ventricular externa, derivação ventricular peritoneal, cânula de traqueostomia, sonda de gastrostomia, cateter de diálise peritoneal, cateter umbilical, dreno de mediastino, dreno de tórax, cateter venoso central e cateter central de inserção periférica, todos de uso neonatal. Os domínios avaliados forma a estética, resistência, uso para ensino, questão sensorial, referências anatômicas e relevância. Todos os dispositivos propostos obtiveram mais de $98,1 \%$ de nota, sendo que na avaliação geral o modelo completo obteve 99,3\%. Conclusão: dada à complexidade do tema e não havendo disponível no mercado um modelo com as características desenvolvidas, o objetivo proposto foi alcançado, a validação e patenteamento pelo Núcleo de Inovação e Transferência de Tecnologia de um novo conceito de manequim neonatal, com oportunidade de inovação, onde o teste de validação permitiu inferir boa aceitação do modelo na prática clínica, mostrando-se uma alternativa efetiva e viável para o ensino do manejo de neonatos que possuam dispositivos, que foram implantados cirurgicamente.
\end{abstract}

Palavras-chave: Manequins; Segurança do paciente; Treinamento por simulação; Unidades de Terapia Intensiva Neonatal; Dispositivos médicos.

\footnotetext{
Abstract

Objective: to develop and validate a neonatal mannequin for skills training in handling the neonate with surgically implanted invasive devices. Method: this is a descriptive quantitative research, with the participation of 21 experts in the process of evaluating the mannequin for validation, respecting all ethical aspects involving research of this nature. To assess the didactic value of the developed product, a validation test was proposed, consisting of experimentation with the model, application of a self-instructional questionnaire containing sociodemographic questions and ten Likert-type scale for each device evaluated. Results and discussion: 10 devices were evaluated in the complete model and they were external ventricular shunt, ventricular peritoneal shunt, tracheostomy cannula, gastrostomy tube, peritoneal dialysis catheter, umbilical catheter, mediastinal drain, chest drain, central venous catheter, catheter peripherally inserted center, all for neonatal use. The domains evaluated form aesthetics, strength, use for teaching, sensory issue, anatomical references and relevance. All of the proposed devices obtained more than
} 
98.1\% of grade, and in the general evaluation of the complete model it obtained $99.3 \%$. Conclusion: given the complexity of the topic and there being no model available on the market with such developed characteristics, the proposed objective was achieved by the Innovation and Technology Transfer Center validation and patenting of a new concept of neonatal mannequin, with opportunity for innovation, where the validation test allowed infer good acceptance of the model in practice, proving to be an effective and viable alternative for teaching the management of newborns who have devices that were surgically implanted.

Keywords: Dummies; Patient safety; Simulation training; Neonatal Intensive Care Units; Medical devices.

\begin{abstract}
Resumen
Objetivo: desarrollar y validar un maniquí neonatal para el entrenamiento de habilidades en el manejo del neonato con dispositivos invasivos implantados quirúrgicamente. Método: se trata de una investigación cualitativo descriptiva, con la participación de 21 expertos en el proceso de evaluación del maniquí para su validación, respetando todos los aspectos éticos que involucran investigaciones de esta naturaleza. Para evaluar el valor didáctico del producto desarrollado, se propuso una prueba de validación, consistente en la experimentación con el modelo, la aplicación de un cuestionario de autoinstrucción que contiene preguntas sociodemográficas y diez escala tipo Likert para cada dispositivo evaluado. Resultados y discusión: se evaluaron 10 dispositivos en el modelo completo y fueron: derivación ventricular externa, derivación peritoneal ventricular, cánula de traqueotomía, sonda de gastrostomía, catéter de diálisis peritoneal, catéter umbilical, drenaje mediastínico, drenaje torácico, catéter venoso central, catéter de inserción periférica central, todo para uso neonatal. Los dominios evaluados forman estética, fortaleza, uso para la enseñanza, tema sensorial, referencias anatómicas y relevancia. Todos los dispositivos propuestos obtuvieron más del 98,1\% de nota, y en la evaluación general del modelo completo obtuvo el 99,3\%. Conclusión: dada la complejidad del tema y al no existir en el mercado un modelo con características tan desarrolladas, el objetivo propuesto se logró mediante la validación y patentamiento Centro de Innovación y Transferencia de Tecnología de un nuevo concepto de maniquí neonatal, con oportunidad de innovación, donde se realizó la prueba de validación Permitió inferir una buena aceptación del modelo en la práctica, demostrando ser una alternativa eficaz y viable para la enseñanza del manejo de los recién nacidos que tienen dispositivos implantados quirúrgicamente.
\end{abstract}

Palabras clave: Maniquíes; Seguridad del paciente; Entrenamiento de simulación; Unidades de Cuidados Intensivos Neonates; Dispositivos médicos.

\title{
1. Introdução
}

O aumento dos casos de prematuridade, registrados nos últimos anos em Unidades de Terapia Intensivas Neonatais (UTIN) (SBP, 2019), tem exigido das equipes, um preparo que sustente a sua complexidade quanto aos cuidados, normas e rotinas, onde diversas vezes os recém-nascidos ficam expostos a possibilidade de eventos adversos refletindo a complexidade desse ambiente terapêutico (Chopra, et al., 2015). Sendo assim, a assistência deve ser realizada de forma segura reduzindo ao mínimo aceitável os riscos associados aos cuidados, onde o cuidado seguro traz diversas temáticas importantes, uma delas diz respeito ao manuseio propriamente dito, com atenção quanto ao perfil desses neonatos que fazem uso de dispositivos invasivos para realização de suporte hídrico e medicamentoso, suporte ventilatório, neurológico e outros que são muitas vezes implantados através de pequenos procedimentos cirúrgicos beira leito (Lui, et al., 2018).

Alguns estudos abordam diretamente esse tema e evidenciam as limitações no manejo de alguns desses dispositivos, como os cateteres centrais de inserção periférica (PICC) (Lui, et al., 2018), assim como outros dispositivos que são facilmente encontrados nesse ambiente como os sistemas de drenagem torácica, sistemas de derivação ventricular externa, cateteres de diálise peritoneais neonatais, cateteres venosos centrais, sondas de gastrostomia e traqueostomias, porém não há foco nos cuidados básicos de rotina do neonatal e sim com os dispositivos. Dentre as dificuldades mais comumente encontradas para a manutenção desse dispositivo encontra a perda acidental em banho, trocas de curativo, manipulação para posicionamento terapêutico entre outros (Krein, et al., 2015).

Muito além de discutir as indicações de todo e qualquer dispositivo é necessário entender o cuidado de manutenção do mesmo para garantir a segurança do recém-nascido gravemente enfermo e, para isso, torna-se necessário formular estratégias e recomendações específicas para o segmento neonatal (Lui, et al., 2018). Pensar em estratégias para evitar perdas ou manipulações inadequadas dos neonatos que possuem dispositivos, incentivando o trabalho em equipe, reduzindo disparidades 
no atendimento, com engajamento em esforços na melhoria da qualidade de assistência, onde o manuseio por vários profissionais e o prolongado período de internação constituem elementos que elevam a exposição à falha (Baladez, 2009). Essas falhas geram aumento dos custos hospitalares, além do aumento da incidência de infecções relacionadas à assistência a saúde, com consequente aumento do uso de antibióticos, tempo de internação hospitalar, morbidade e mortalidade em UTIN (Lanzillotti, 2015; Pires \& Lima, 2019).

Existem no mercado hoje, manequins não humanos de simulação para treino do ato de implantação de diversos dispositivos, com foco na questão do ganho de habilidades para sua inserção e implementação. Com base na crescendo necessidade de melhora da segurança do cuidado, há a necessidade de trabalhar na atenção do manejo de neonatos que possuam estes dispositivos, após implantação, em um segundo momento do cuidado, agora com foco na manipulação por diversos profissionais que compõe a equipe multiprofissional, para evitar perdas desnecessárias aumentando assim risco de infecção, risco da repetição do procedimento, aumento do custo da internação com prolongamento dos dias de UTIN e risco inerentes de perdas durante manobras comuns na rotina dos atendimentos multiprofissionais.

Neste contexto científico e assistencial, visando a busca por soluções na qualidade e melhoria do cuidado prestado ao paciente neonatal criticamente enfermo, a pesquisa teve como objetivo desenvolver e validar um simulador neonatal para treinamento de habilidades no manuseio do neonato com dispositivos invasivos implantados cirurgicamente.

\section{Método}

Os aspectos éticos foram respeitados em todas as etapas do estudo, com aprovado pelo Comitê de Ética da Universidade do Estado do Pará (UEPA), CAAE: 32823720.0.0000.8607. O estudo envolveu as seguintes etapas: desenvolvimento do produto, processo de validação, análise estatística dos dados obtidos, depósito da patente do modelo de utilidade e divulgação dos resultados.

\subsection{Desenho e delineamento do estudo e aspectos éticos}

Foi desenvolvido um estudo caracterizado como quantitativo descritivo em que foram utilizados métodos quantitativos para análise de dados, não intervencionista, com financiamento próprio (Lacerda \& Costenaro, 2016; Caselato, 2019). A pesquisa caracterizou-se por algumas etapas: pesquisa bibliográfica acerca do tema e busca nas bases de dados de patentes, desenvolvimento do produto inicialmente como protótipo, com aperfeiçoamento contínuo, até que o simulador estivesse preparado para o processo de validação.

$\mathrm{Na}$ fase de validação foram convidados para inicialmente para 10 juízes especialistas com atuação assistencialista hospitalar em terapia intensiva neonatal e / ou docente na área da saúde com foco em atendimento neonatal contando com profissionais de saúde de distintas formações: médicos, enfermeiros, fisioterapeuta e fonoaudiólogos.

A seleção foi realizada por análise do Currículo Lattes e comprovação de vínculo com unidade hospitalar pelos cadastrados no CNES, com atuação mínima de 05 anos em UTIN, título de especialista na área, atuação assistencialista hospitalar neonatal. Por método de bola de neve (Dewes, 2013) foram incluídos mais 14 juízes especialistas que apresentavam critérios de inclusão onde após o primeiro contato e declarado o aceite para participar, esta foi formalizada pela assinatura do Termo de Consentimento Livre e Esclarecido.

\subsection{Fase de desenvolvimento}

$\mathrm{Na}$ fase de desenvolvimento o planejamento do produto foi realizado e a elaboração de um protótipo. No projeto informacional foram definidas metas e especificações do produto que posteriormente iriam direcionar como parâmetros para exigência de qualidade e padrão. No projeto conceitual foi determinada a funcionalidade da criação, buscando arquitetar 
soluções e elaborar um plano de processo de fabricação só então foi realizado o projeto detalhado com escolha de materiais que seriam adaptados em um boneco comercial siliconado com aspecto mais próximo de um recém-nascido real, sem aspecto de malformações congênitas, pesando $1.300 \mathrm{~g}$, prematuro, considerando os possíveis riscos, os materiais apropriados, a viabilidade de técnicas de produção, os equipamentos exigidos no processo e o dimensionamento adequado para atender aos requisitos e só então foi conduzida a preparação e produção, análise de valores, gerenciamento da durabilidade, revisão e aprimoramento, além da redução de custos. Sendo então atingido o produto final.

O desenvolvimento do manequim utilizou recursos de baixo custo e tecnologia, realizado pela equipe sem custo de mão-de-obra, intitulado ConectNeo, com slogan para marketing: "conecte-se nessa ideia - uma solução para treinamento de habilidades no manuseio do neonato com dispositivos invasivos"; sendo finalizado para etapa de validação após 02 protótipos, onde o protótipo representa a tangibilização de uma ideia, do abstrato para o físico, reduzem as incertezas do projeto, pois é nessa fase onde elimina-se alternativas inviáveis e portanto, auxiliam no desenvolvimento de uma solução final mais assertiva para o produto proposto (Hübner, 2017). O desenvolvimento de um produto é um processo complexo, portanto é necessária a visão de diferentes áreas de conhecimento, nesse projeto foram envolvidos uma equipe multidisciplinar representada por: enfermeira, fisioterapeuta, administrador que avaliou potencial de mercado e profissional com formação em juiz de direito que possuía habilidade em prototipagem rápida. O manequim para validação proposto foi constituído por um boneco de corpo inteiro não eletrônico, com locais específicos anatomicamente para conexão dos dispositivos que simulam dispositivos implantados cirurgicamente rotineiramente em UTIN como pode ser visto na Figura 1. Foram desenvolvidos 10 pontos de encaixe de dispositivos adaptados para tal finalidade, sendo eles: derivação ventricular externa, derivação ventricular peritoneal, cânula de traqueostomia, sonda de gastrostomia, cateter de diálise peritoneal, cateter umbilical, dreno de mediastino, dreno de tórax, cateter venoso central, cateter central de inserção periférica, todos de uso neonatal.

Figura 1. Modelo da tese.

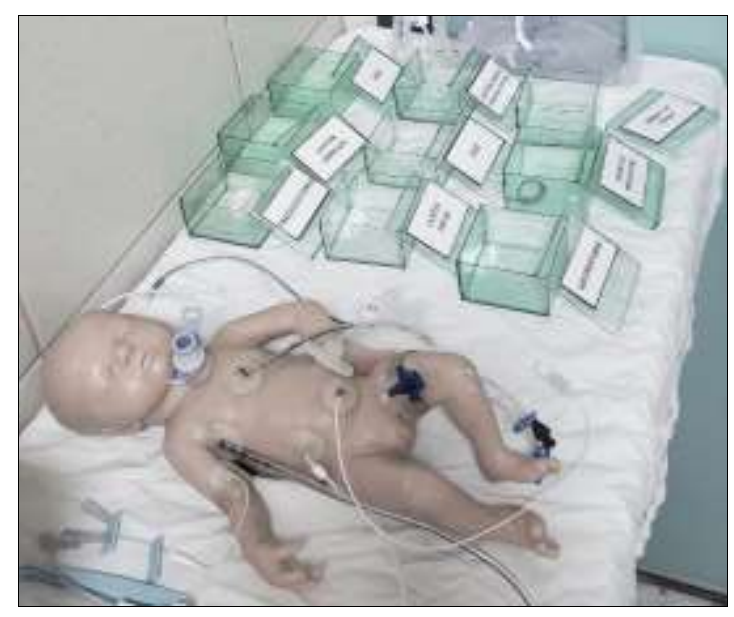

Fonte: Arquivo da tese.

\subsection{Fase de validação}

Para avaliação do valor didático do produto desenvolvido foi proposto um teste de validação que consiste em experimentação do modelo, aplicação de um questionário autoinstrutivo contendo questões sociodemográficas e escala tipo Likert para cada dispositivo avaliado, totalizando 10 dispositivos no manequim. Cada questionário continha 09 domínios e questões objetivas sobre a nota atribuída ao produto, sugestões de melhoria e no início do questionário questões demográficas.

Para validação do simulador desenvolvido, foram utilizadas dez escalas do tipo Likert, adaptadas a cada um dos procedimentos que compõem o protótipo. Foi integrado ao instrumento avaliativo, um domínio sobre os dados demográficos 
dos juízes participantes do estudo. Os dados sociodemográficos continham: sexo, idade, profissão, tempo de atuação profissional, vínculo atual com IES e tempo de docência. As escalas de Likert adaptadas possuíam nove afirmativas, que avaliavam os domínios do simulador quanto: a avaliação estética, a resistência, ao ensino, a questão sensorial, as referências anatômicas, a relevância para prática profissional, a presença de defeitos, a habilidades e por último a funcionalidade. Os juízes tinham disponível, cinco níveis de concordância para escolha, assim denominados: discordo totalmente, discordo parcialmente, nem concordo e nem discordo, concordo parcialmente e concordo totalmente. $\mathrm{O}$ instrumento da pesquisa possuía ainda: nota atribuída ao modelo e espaço para sugestões de melhoria pontuadas pelo participante.

O teste de validação foi iniciado e concluído em dezembro de 2020, sendo a amostra dividida em quatro grupos de juízes especialistas: sete médicos pediatras, sete enfermeiros, cinco fisioterapeutas e dois fonoaudiólogos. Os testes foram padronizados e realizados em sessões individuais com cada participante em seu local de trabalho, em sala reservada, durante o período que o avaliador dispunha e tomando devidas medidas de biossegurança quanto a Covid-19. Ao especialista foi entregue uma mala de transporte fechada contendo o modelo de treinamento para avaliação, sendo realizada explicação sobre o processo de avaliação e orientado que a verificação de cada dispositivo fosse realizada separadamente seguindo a ordem céfalo-caudal para facilitar a compreensão e a prática, além de dinamizar posteriormente as respostas do instrumento avaliativo do modelo (questionário contendo as escalas do tipo Likert) proposto. Em seguida à execução do procedimento de avaliação de cada dispositivo então, era dada opção ao participante de preencher a escala de Likert daquele dispositivo, assim evitando confundir as respostas caso fosse realizado somente ao final da avaliação dos 10 dispositivos. Ao término o participante lacrava em envelope próprio protegendo o anonimato, que era entregue ao pesquisador, para posterior análise dos resultados.

\subsection{Depósito de patente do Modelo de Utilidade.}

Para viabilização da ideia do modelo de treinamento, foi primeiramente realizado um levantamento de patentes no mercado, sendo consultadas as seguintes bases de dados: Instituto Nacional de Produção Intelectual (INPI), European Patent Office (EPO), United States Patent and Trademark Office's (USPTO), China Trade Mark Office (CTPO), Japan Patent Office (JPO), Google patente, não sendo encontrado nenhum modelo patenteado com todos esses dispositivos desenvolvidos em um só modelo, sendo 04 deles totalmente inéditos no produto deste estudo.

Após finalização do produto foi dada então entrada no pedido de patente no Núcleo de Inovação e Transferência de Tecnologia (NITT) da UEPA, com depósito de patente de modelo de utilidade realizado em dezembro de 2020 com processo de $\mathrm{n}^{\circ}$ BR 2020200260559 e registro de marca no Instituto Nacional da Propriedade Industrial (INPI) com $\mathrm{n}^{\circ}$ protocolo 850210011541.

\subsection{Análise Estatística}

Após a coleta e tabulação de dados em planilhas eletrônicas, utilizando o software Excel 2016, os mesmos formam analisados estatisticamente, com nível de significância $\alpha=0,05$. Os resultados foram apresentados sob a forma de tabelas e gráficos no Microsoft Excel®. Os dados numéricos foram analisados mediante uma estatística descritiva.

\section{Resultados}

Dos 24 juízes especialistas convidados, somente 03 (1,24\%) não participaram, totalizando um $\mathrm{N}$ de 21 participantes. Entre eles $07(33,3 \%)$ médicos pediatras, 02 (9,5\%) fonoaudiólogas, 05 (23,8\%) fisioterapeutas, $07(33,3 \%)$ enfermeiros. Todos com expertise comprovada na área do estudo (Tabela 1). A validação foi desenvolvida em um único encontro, com duração média de uma hora, no qual foi feita a apresentação do projeto de pesquisa, seguida pela exploração do modelo. Os itens avaliados pelos especialistas no processo de validação estão descritos detalhadamente na Tabela 2 sendo os domínios 
avaliados quanto a avaliação estética (se simula a realidade), quanto a resistência (se o modelo possue robustez), quanto ao ensino (se é adequado para uso em aulas práticas), quanto a questão sensorial (se reproduz as condições reais do procedimento proposto), quanto as referências anatômicas (se possui todas as referências anatômicas contempladas no manuseio de cada dispositivo proposto), em relação a relevância (se possui relevância para prática profissional), em relação a presença de defeitos (se apresenta defeitos que são incompatíveis para o treinamento de cuidados com modelo), quanto a habilidades (se poderá será utilizado nos treinamentos para a aquisição de habilidades técnicas) e por último quanto a funcionalidade (se pode ser considerado funcional). Na Tabela 3 são apresentados os resultados da avaliação geral dos dispositivos presentes no modelo com critérios para validação dos mesmos, bem como número de juízes que participou do processo de cada um e porcentagem alcançada. Como pode ser visto na tabela 3, cinco dispositivos foram analisados por 19 dos 21 juízes onde profissionais fonoaudiólogos se autodeclaram não aptos no momento para analisar itens relacionados ao sistema de derivação ventricular externa, derivação peritoneal, dreno de tórax, drenagem mediastinal e dispositivo de diálise peritoneal, por tais dispositivos não fazerem parte de suas rotinas de trabalho.

Tabela 1 - Caracterização da amostra do estudo, Marabá - PA, 2020.

\begin{tabular}{|c|c|c|}
\hline Variáveis & $\mathbf{N}$ & $\%$ \\
\hline \multicolumn{3}{|l|}{ Sexo } \\
\hline Masculino & 1 & $4,70 \%$ \\
\hline Feminino & 20 & $95,20 \%$ \\
\hline \multicolumn{3}{|l|}{ Idade } \\
\hline $29-39$ anos & 11 & $52,30 \%$ \\
\hline $40-54$ anos & 10 & $47,60 \%$ \\
\hline \multicolumn{3}{|l|}{ Profisssão } \\
\hline Médico & 7 & $33,30 \%$ \\
\hline Enfermeiro & 7 & $33,30 \%$ \\
\hline Fisioterapeuta & 5 & $23,80 \%$ \\
\hline Fonoaudiólogo & 2 & $9,50 \%$ \\
\hline \multicolumn{3}{|l|}{ Tempo de atuação } \\
\hline $6-15$ anos & 13 & $61,90 \%$ \\
\hline $16-25$ anos & 4 & $19 \%$ \\
\hline $26-35$ anos & 4 & $19 \%$ \\
\hline \multicolumn{3}{|l|}{ Vínculo atual com IES } \\
\hline Sim & 6 & $28,50 \%$ \\
\hline Não & 15 & $71,40 \%$ \\
\hline \multicolumn{3}{|l|}{ Tempo de docência } \\
\hline $1-5$ anos & 6 & $75 \%$ \\
\hline $6-15$ anos & 1 & $12,50 \%$ \\
\hline 29 anos & 1 & $12,50 \%$ \\
\hline
\end{tabular}

Fonte: Santos et al. (2021). 
Tabela 2 - Apresentação dos domínios avaliados para cada dispositivo proposto no modelo.

DISPOSITIVOS ANALISADOS NO MODELO

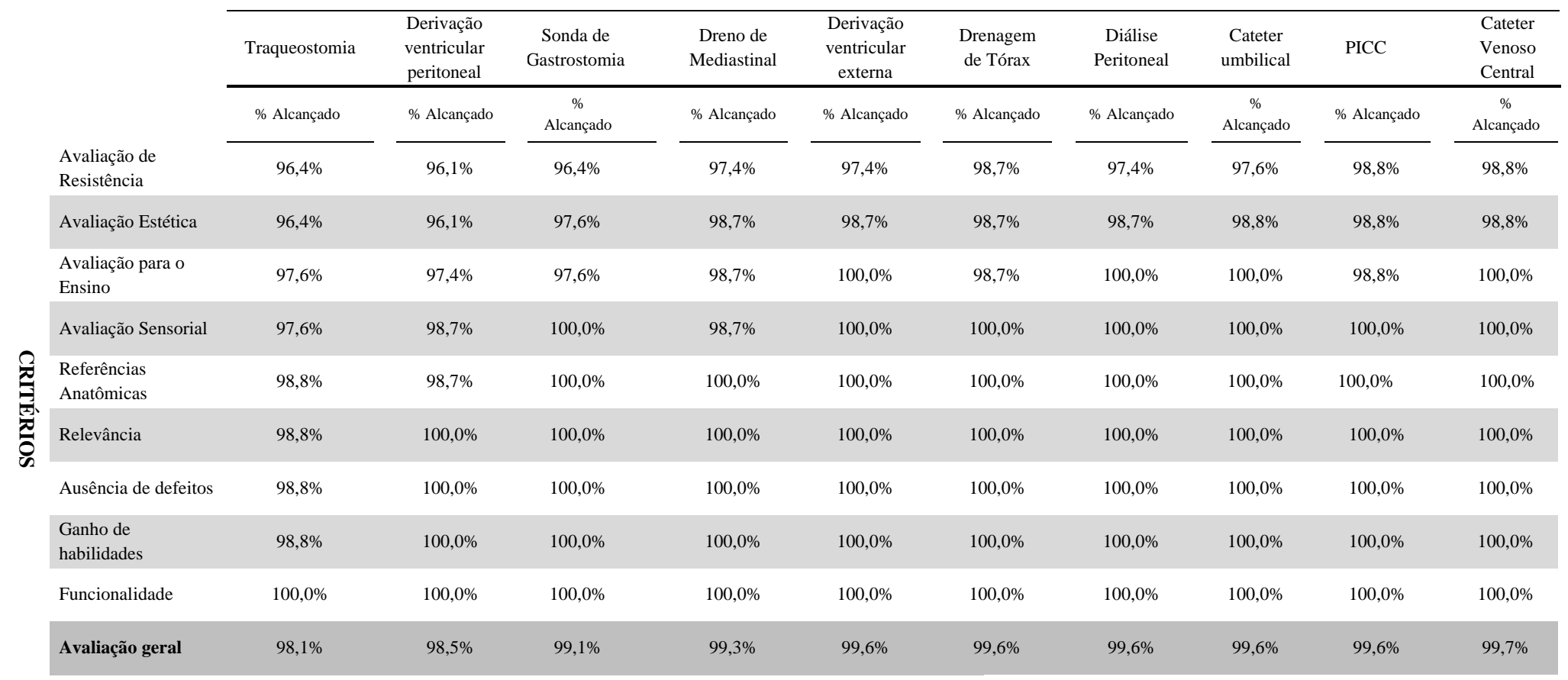

Legenda: DVP (Derivação ventricular peritoneal), DVE (Derivação ventricular externa), CUV (cateter umbilical venoso), PICC (cateter central de inserção periférica)

Tabela 3 - Avaliação geral dos dispositivos presentes no modelo com critérios para validação.

\begin{tabular}{|c|c|c|c|c|c|}
\hline Dispositivos & $\mathbf{N}^{o}$ Juízes & \% Alcançado & & & \\
\hline Traqueostomia & 21 & $98,1 \%$ & & & \\
\hline Derivação Peritoneal & 19 & $98,5 \%$ & \multicolumn{3}{|c|}{ Critérios de validação } \\
\hline Sonda de Gastrostomia & 21 & $99,1 \%$ & Porcentagem & Classificação & Conceito \\
\hline Drenagem de Mediastino & 19 & $99,3 \%$ & \multirow{2}{*}{$0 \%$ a $25 \%$} & \multirow{2}{*}{ Ruim } & \multirow{2}{*}{ Não validado } \\
\hline Derivação Ventricular Externa & 19 & $99,6 \%$ & & & \\
\hline Drenagem de Tórax & 19 & $99,6 \%$ & \multirow{2}{*}{$25.1 \%$ a $50 \%$} & \multirow{2}{*}{ Regular } & \multirow{2}{*}{ Não validado } \\
\hline Diálise Peritoneal & 19 & $99,6 \%$ & & & \\
\hline Cateter Umbilical & 21 & $99,6 \%$ & \multirow{2}{*}{$50.1 \%$ a $75 \%$} & \multirow{2}{*}{ Bom } & \multirow{2}{*}{$\begin{array}{c}\text { Validado com } \\
\text { ressalvas }\end{array}$} \\
\hline PICC & 21 & $99,6 \%$ & & & \\
\hline Cateter Venoso Central & 21 & $99,7 \%$ & \multirow{2}{*}{$\begin{array}{c}75.1 \% \text { a } \\
100 \%\end{array}$} & \multirow{2}{*}{ Excelente } & \multirow{2}{*}{ Validado } \\
\hline Avaliação Geral & & $99,3 \%$ & & & \\
\hline
\end{tabular}

Fonte: Santos et al. (2021).

\section{Discussão}

Segundo Baladez (2009) o conhecimento alcançado partindo de experiências simuladas, solidifica-o tornando-o palpável, observável, evidente pela possibilidade de utilizar-se de mais de um sentido, o que pode ser considerado como um recurso didático útil para atingir um objetivo de ensino. Nenhuma instituição, seja ela de formação educativa, como instituições de ensino superior ou instituições de atendimento hospitalar podem negligenciar o fato de que é essencial a busca constante de oportunidades de aprendizagem, propiciando atualizações profissionais, reciclagens, treinamentos específicos e 
capacitações internas difundindo as melhores práticas de cuidados. Para alguns autores o manejo adequado do neonato tornase necessário e os constantes incentivos à qualificação profissional sempre amparadas em bases científicas (Chopra, et al., 2015).

Pensando na possibilidade de evitar desconfortos e risco ao paciente, quando se trabalha com melhoria das curvas de aprendizado e redução dos custos chama atenção quanto ao uso de manequim para ensino, além das demandas éticas existe a vantagem didática no uso de modelos de treinamento em relação a possibilidade de análise dos erros cometidos em ambiente simulado, deixam de ser onerosos para terceiros e passando a lhe ser proveitosos, permitindo identificar lacunas no conhecimento e/ou habilidades (Temperly, et al,, 2018; Balbino, et al., 2020).

O modelo desenvolvido nesse estudo é classificado como de baixa fidelidade, caracterizado pela integração de pouca tecnologia e destinado à aprendizagem e prática de habilidades simples (Silva \& Pereira, 2018). Quanto ao termo fidelidade, o mesmo descreve o realismo da experiência e não está relacionado a robotização, sendo que fidelidade e tecnologia devem ser analisadas separadamente (Walsh, 2012).

Nesse estudo, de maneira geral, as respostas demonstraram a boa aceitação do modelo, tanto da experiência quanto da funcionalidade do mesmo. A amostra de mais de uma instituição, com tempo de experiência ampla o que demonstra maior confiança nos dados avaliados, em outros estudos autores evidenciam as limitações focadas em pequenas amostras e associada a homogeneidade, com faixa relativamente estreita sendo um fator limitante para estudos nessa área (Barsness, et al., 2013) onde os resultados esperados muitas vezes estão correlacionados com os níveis de experiência do participante (Hamilton, et al,, 2013).

Barsness et al. (2013) desenvolveram um simulador de atresia esofágica toracoscópica com fístula traqueoesofágica, utilizaram o mesmo processo de validação com amostra de 11 especialistas, preencheram um relatório com escala de classificação, variando de 1 (não sei) a 5 (altamente realista), avaliando a relevância, atributos físicos, realismo dos materiais e capacidade de executar tarefa no simulador.

Em estudo realizado por Christie e Kristen em 2016, os mesmos demostraram um levantamento de opções de baixo custo para simulação e treinamento em neonatologia para o ensino de habilidades clínicas e procedimentos de intubação neonatal, traqueostomia, inserção de acesso venoso central em diversos pontos anatômicos, inserção de dreno torácico, pericardiocentese, kits de simulam incluindo gastrosquise, onfalocele, mielomeningocele e distensão abdominal. Os autores relatam que não há evidências suficientes para determinar se simulação de alta fidelidade é melhor do que simulação de baixa fidelidade.

Temperly et al. em 2018, desenvolveram e validaram um simulador de traqueostomia de baixo custo em adulto, existindo atualmente diversos modelos comerciais no mercado tanto adulto quanto pediátrico.

Quanto ao manequim com dispositivos de derivação ventricular externa (DVE) e derivação ventricular peritoneal proposto neste estudo, são descritos na literatura somente em modelo adulto com simulador virtual de drenagem ventricular externa para dispositivo móvel com finalidade de melhorar as habilidades dos residentes na realização da técnica de inserção da DVE (Morone, et al., 2017) e nenhum físico foi identificado. Existe no mercado um simulador pediátrico para simulação do ato cirúrgico de neuroendoscopia e cranioestenose (Caselato, 2019) porém nenhum modelo anatômico abordando o cuidado com a derivação ventricular peritoneal foi identificado na literatura atual. O modelo desenvolvido tem objetivo de criar ambiente propicio para desenvolver rotinas de cuidados com bebê que possuam DVE e / ou derivação ventricular peritoneal evitando que os profissionais tenham contato com esses dispositivos somente quando houver na unidade neonatal um caso, visto que não é procedimento corriqueiro na grande maioria das unidades neonatais. 
Tanaka et al. publicaram, em 2021, um manual voltado para enfermagem sobre cuidados com DVE, evidenciando a necessidade de materiais voltado para cuidados de enfermagem no posicionamento, cuidados de rotina, no transporte e nas intercorrências.

Quanto a modelos para inserção do tubo torácico o que se sabe é que profissionais que cuidam de bebês gravemente doentes precisam adquirir competência na inserção de tubos torácicos para pneumotórax, sendo disponíveis no mercado modelos para treinar a inserção de tubo torácico de baixo e alto custo, (Ashish \& Ramasethu, 2013; Christie, et al., 2016) não sendo o foco o pós-operatório e cuidados no manuseio do bebê, como banho, mudanças de decúbito, realizações de técnicas específicas, por exemplo, quanto a manobras fisioterápicas, com estudos focados nessa divulgação para o meio cientifico. Quanto ao dreno de mediastino não houve evidência de manequim disponível no mercado com esse sistema em simuladores neonatais e / ou adulto com esse dispositivo.

Biazi et al. em 2009 desenvolveram um dispositivo de equipo de diálise peritoneal pediátrico totalmente fechado de fácil manuseio com parceria com Setor de Bioengenharia da Braile Biomédica Indústria, Comércio e Representações S/A®. Não havendo atualmente, nas bases de patentes nenhum modelo com cateter específico de diálise exclusivamente neonatal que possa ser utilizado para treinar o manuseio do esquema do sistema de diálise peritoneal e a gestão do cuidado de enfermagem, fisioterapia mais específicos com o neonato, que esteja em procedimento dialítico, como por exemplo, um transporte intrahospitalar do bebê em diálise e manuseio de rotina do neonato com cateter. $\mathrm{O}$ modelo proposto nesse estudo abrange essa temática de grande relevância, apresentando na avaliação geral excelente avaliação dos juízes especialistas, sem ressalvas e descrições de melhorias.

Quanto ao dispositivo de cateterismo umbilical, grandes empresas desenvolveram e aperfeiçoaram ao longo dos anos, diversos modelos de baixo custo para treinar o procedimento médico e de enfermagem para passagem de cateter umbilical e assim discutir as indicações, contraindicações e complicações associadas ao procedimento, demonstrar a fixação adequada. Em 2018 um grupo de pesquisadores do Hospital Kings County do Departamento de Medicina de Emergência Pediátrica, desenvolveram um cordão umbilical usando cateteres de sucção para representar as artérias umbilicais e tubo de oxigênio da cânula nasal representando a veia umbilical, suspensos em um base de silicone e enrolada em forma de tronco para simular o cordão umbilical e utilizaram uma boneca de brinquedo com um pequeno orifício circular no abdome oco para fixar esse cordão. Uma vez montado, foi utilizado como instrumento de tarefas em sessões de procedimento instrucional (Caitlin \& Linda, 2018).

Várias empresas produzem cordões umbilicais para comercialização com finalidade de treino, modelos com local para treino de inserção do cateter e estudos de desempenho da inserção da linha umbilical (Sawyer, 2009; Christie, et al., 2016). O modelo proposto nesse estudo abrange este dispositivo que apresentou avaliação excelente, com objetivo de tornar o modelo o mais completo possível.

A cerca do PICC, Freitas NF et al. em 2011 abordaram a necessidade de discussões sobre intervenções para minimizar os eventos adversos relacionados ao PICC como perda acidental durante manipulação do recém-nascido, questões relacionadas a enfermagem como cuidados com conexões durante o banho e outros. Existem hoje no mercado alguns modelos com diversos caraterísticas nessa temática. Modelos na categoria neonatal simulam bebês de até 28 dias, com idade gestacional de nascimento simulando entre $24 \mathrm{~s}$ e até $40 \mathrm{~s}$, com diversidade de pesos que variam de $600 \mathrm{~g}$ até $3 \mathrm{~kg}$, com marcas mais encontradas da CAE Healthcare, SIMCharacters, Limbs and Things, 3B Scientific, Life/form ${ }^{\circledR}$, Gaumard, Laerdal, Anatomic, Simulacare, Simulaids e REALCARETM. Dentro das caraterísticas que o produto da tese propõe foram encontrados modelos que possuem local de traqueostomia, ósteo de gastrostomia, locais periféricos de cateteres central venosos, cordão umbilical para cateterismo, inserção de linha PICC e drenagem torácica na região hemiaxilar, porém nenhum reunindo todos os dispositivos como a proposta desta pesquisa. 
Para Silva et al. (2018) a complexidade do local de trabalho exige o uso de modelos e simulação como recurso, uma ferramenta útil para identificação de pontos críticos na execução das habilidades e reforço do conhecimento principalmente para profissionais com menos experiencia. Estudos específicos sobre transporte de pacientes pediátricos graves também reportam sobre a importância de treinamentos baseados em simulação utilizando muitas vezes manequins de alta fidelidade para melhora na confiança, planejamento e organização do equipamento de transporte e estratégias de comunicação (Murdoch, et al., 2019).

O modelo proposto, validado e patenteado, busca unir em um só simulador não humano neonatal, dez dispositivos mais comuns utilizados em um neonato grave em unidades de terapia intensiva, abrindo leque de possibilidades de treinamento com diversas oportunidades de vivenciar na prática o pegar, tomar, manobrar, cuidar, podendo o profissional encontrar tudo em um instrumento único, dinamizando o serviço de educação continuada.

Uma limitação específica deste estudo foi relacionada a Pandemia da Covid 19, onde os pesquisadores modificaram a metodologia do estudo e adequaram-se quanto as recomendações da Organização Mundial de Saúde relacionadas a prevenção e proteção de todos os envolvidos na pesquisa, não havendo tempo hábil por parte dos pesquisadores para ampliar o $\mathrm{N}$ amostral devido as limitações impostas pela limitação de atividades presencias nas instituições de ensino superior no período de coleta de dados.

\section{Considerações Finais}

Dada à complexidade do tema e não havendo disponível no mercado um modelo de treinamento com tais características desenvolvidas, o objetivo proposto foi alcançado havendo a validação e patenteamento de um novo conceito de simulador neonatal, com oportunidade de inovação, tendo como final um produto físico cujas características dão condições de, em projetos futuros, serem reproduzidos comercialmente, onde o teste de validação permitiu inferir boa aceitação do modelo na prática, mostrando-se uma alternativa efetiva e viável para o ensino do manejo do neonato, que possuam dispositivos que foram implantados cirurgicamente.

\section{Contribuições dos autores}

Todos os autores fizeram contribuições substanciais para concepção, ou delineamento, ou aquisição, ou análise ou interpretação de dados; e redação do trabalho ou revisão crítica; e aprovação final da versão para publicação.

\section{Referências}

Ashish, O. G. \& Ramasethu, J. (2014). An innovative nonanimal simulation trainer for chest tube insertion in neonates. Pediatrics,134, e798. doi:10.1542/peds.2014-0753

Baladez, F. (2009). O passado, o presente e o futuro dos simuladores. Fasci-Tech: Periódico Eletrônico da FATEC, 1(1):29-40.

Balbino, A. C., Silva, A. N. S., \& Queiroz, M. V. O. (2020). O impacto das tecnologias educacionais no ensino de profissionais para o cuidado neonatal. Revista Cuidarte, 11(2): e954. doi:10.15649/cuidarte.954.

Barsness, K. A, Rooney, D. M., \& Davis, L. M. (2013). Collaboration in simulation: The development and initial validation of a novel thoracoscopic neonatal simulator. Journal of Pediatric Surgery, 48:1232-1238. https://pubmed.ncbi.nlm.nih.gov/23845612/

Biazi, A. P. P., Crotti, U. A., Braile, D. M., Oliveira, M. A. B., Costa, J. G. S., \& Cardoso, L. M. (2009). Desenvolvimento e avaliação da esterilidade de sistema de diálise peritoneal pediátrico fechado. Rev Bras Cir Cardiovasc, 24(1): 64-67. doi: https://doi.org/10.1590/S0102-76382009000100012

Caitlin, W. F., \& Linda, F. (2018). Low Cost Task Trainer for Neonatal Umbilical Catheterization. Electronically Published, 3(3):133-40. 10.21980/J8HW6G

Caselato, G. C. R. (2019). Uso de simuladores realísticos em neurocirurgia pediátrica. Faculdade de Medicina, 1-129. https://doi.org/10.11606/T.5.2019.tde$01072019-144748$ 
Chopra, V., Flanders, A. S., Saint, S., Woller, S. C., O'Grady, N. P., Safdar, N. et al. (2015). The Michigan appropriateness guide for intravenous catheters (MAGIC): results from a multispecialty panel using the RAND/UCLA appropriateness method. Ann Intern Med, Sep 15,163(6 Suppl):S1-40. https://doi.org/10.7326/M15-0744

Christie, J. B., \& Kristen, M. G. (2016). Cost-effective and low-technology options for simulation and training in neonatology. Seminars in Perinatology, 1-7. doi: http://dx.doi.org/10.1053/j.semperi.2016.08.008

Dewes, J. O. (2013). Amostragem em bola de neve e respondente-drive sampling: uma descrição dos métodos. Universidade Federal do Rio Grande do Sul (Monografia), p. 53. https://www.lume.ufrgs.br/handle/10183/93246

Freitas, N. F. (2011). A tecnologia social e cuidar na enfermagem: instrumentos necessários para a construção da educação em serviço em saúde em uma Unidade Neonatal de Cuidados Progressivos [dissertação mestrado]. Belo Horizonte: Centro Universitário UNA.

Hamilton, J. B., Pena, V., Haddad, M., \& Clarke, S. (2013). Simulation in the Early Management of Gastroschisis. Simulation in Healthcare, 8 (6): $376-381$. doi: 10.1097/SIH.0b013e31829b3ff9.

Hübner, G. S. S. (2017). Desenvolvimento de um manequim simulador de punção venosa para educação na saúde: da ideia ao protótipo. Dissertação de mestrado do Programa de pós-graduação em Engenharia de Produção - UFRS, 1-103. http://hdl.handle.net/10183/139416

Krein, S. L., Kuhn, L., Ratz, D., \& Chopra, V. (2015). Use of designated nurse PICC teams and CLABSI prevention practices among U.S. hospitals: a surveybased study. J Patient Saf, 10. doi:10.1097/PTS.0000000000000246

Lacerda, R. M., \& Costenaro, R. G. S. (2016). Metodologias da pesquisa para a enfermagem e saúde: da teoria à prática. Porto Alegre: Moriá, 511 p.:il

Lanzillotti, L. S., Seta, M. H., Andrade, C. L. T., Mendes, Jr., \& Vieira, W. (2015). Eventos adversos e outros incidentes na unidade de terapia intensiva neonatal. Ciênc. saúde coletiva [online], 20(3): 937-946. ISSN 1413-8123. 10.1590/1413-81232015203.16912013.

Lui, A. M. L., Zilly, A., França, A. F. O., Ferreira, H., Toninato, A. P. C., \& Silva, R. M. M. (2018). Cuidados e limitações no manejo do cateter central de inserção periférica em neonatalogia. Revista de Enfermagem do Centro-Oeste Mineiro, 8:e1918. 10.19175/recom.v7i0.1918

Melo, R. P., et al. (2011). Critérios de seleção de experts para estudos de validação de fenômenos de enfermagem. Rev Rene, Fortaleza, abr/jun, 12 (2):424-31. http://periodicos.ufc.br/rene/article/view/4254

Morone, P. J., Bekelis, K., Root, B. K., \& Singer, R. J. (2017). Development and Validation of a Mobile Device-based External Ventricular Drain Simulator. Operative Neurosurgery, 13:603-608. doi: 10.1093/ons/opx022

Murdoch, M., Kache, S., McNabb, L., Palmquist, A., Olson, M., \& Sacks, L. (2019). A Novel Simulation Based Method For Training Pediatric \& Neonatal Critical Care Transport Teams. Pediatrics, August, 144-875. doi: https://doi.org/10.1542/peds.144.2_MeetingAbstract.875

Pires, A. B. M., \& Lima, A. F. C. (2019). Custo direto da passagem de cateter central de inserção periférica por enfermeiros. Rev. Bras. Enferm., 72(1):95-101. doi: https://doi.org/10.1590/0034-7167-2018-0250

Ribeiro, V. S., Garbucio, D. C., Zamariolli, C. M., Eduardo, A. A., \& Carvalho, E. C. (2018). Simulação clínica e treinamento para as Práticas Avançadas de Enfermagem: revisão integrativa. Acta Paul Enferm, 31(6):659-66. doi: https://doi.org/10.1590/1982-0194201800090

Sawyer, T., Hara, K., Thompson, M. W., Pharm, D. S. C., \& Berg, B. (2009). Modification of the Laerdal SimBaby to include an integrated umbilical cannulation task trainer. Simulation in Healthcare, 4(3). https://journals.lww.com/simulationinhealthcarebyBhDMf5ePHKav1zE oum1tQfN4a+kJLhEZgb sIHo4XMiOhCywCX1AWnYQp/IIQrHD3YiN3qTGLcvLVeFF55FweqKhGf9seOqSNHlpQUjgMeBbBieWHT8FsgQ== on 07/04/2020.

SBP. (2019). 15,9 mil leitos de internação pediátrica foram fechados no Brasil, nos últimos nove anos. https://www.sbp.com.br/imprensa/detalhe/nid/159-milleitos-de-internacao-pediatrica-foram-fechados-no-brasil-nos-ultimos-nove-anos/

Silva, J. P., Pereira, G. A. Jr., Meska, M. H. G., \& Mazzo, A. (2018). Construction and validation of a low-cost simulator for training patients with diabetes mellitus and/or their caregivers in insulin administration. Escola Anna Nery, 22(3):1-7. 10.1590/2177-9465-EAN-2017-0387

Tanaka, A. K. S. R., et al. (2021). Manual de orientações sobre cuidados de Enfermagem com pacientes em uso de Derivação Ventricular Externa e Monitorização da Pressão Intracraniana. UFRGS, 26 p.: il.

Temperly, K. S., Yaegashi, C. H., Silva, A. M. L., \& Novak, E. M. (2018). Desenvolvimento e validação de um simulador de traqueostomia de baixo custo (Development and validation of a low-cost tracheostomy simulator). Sci Med, 28(1). http://doi.org/10.15448/1980-6108. 2018.1.28845

Temperly, K. S., Yaegashi, C. H., Silva, A. M. L., \& Novak, E. M. (2018). Development and validation of a low-cost tracheostomy simulator. Sci Med, 28(1). http://doi.org/10.15448/1980-6108.2018.1.28845

Walsh, K., \& Jaye, P. (2021). The relationship between fidelity and cost in simulation. Med Educ, 46(12):1226. http://onlinelibrary.wiley.com/doi/ 10.1111/j.1365-2923.2012.04352.x/full 